\title{
Sensing of pulsed radiation with Pyroelectric Detectors
}

Link to publication record in Manchester Research Explorer

\section{Citation for published version (APA):}

Efthymiou, S., \& Krikor, O. (2009). Sensing of pulsed radiation with Pyroelectric Detectors. In Sensors \& their Applications XV IOP Publishing Ltd.

\section{Published in:}

Sensors \& their Applications XV

\section{Citing this paper}

Please note that where the full-text provided on Manchester Research Explorer is the Author Accepted Manuscript or Proof version this may differ from the final Published version. If citing, it is advised that you check and use the publisher's definitive version.

\section{General rights}

Copyright and moral rights for the publications made accessible in the Research Explorer are retained by the authors and/or other copyright owners and it is a condition of accessing publications that users recognise and abide by the legal requirements associated with these rights.

\section{Takedown policy}

If you believe that this document breaches copyright please refer to the University of Manchester's Takedown Procedures [http://man.ac.uk/04Y6Bo] or contact uml.scholarlycommunications@manchester.ac.uk providing relevant details, so we can investigate your claim.

\section{OPEN ACCESS}


Pulsed performance of pyroelectric detectors

This article has been downloaded from IOPscience. Please scroll down to see the full text article.

2009 J. Phys.: Conf. Ser. 178012044

(http://iopscience.iop.org/1742-6596/178/1/012044)

View the table of contents for this issue, or go to the journal homepage for more

Download details:

IP Address: 84.73.14.172

The article was downloaded on 02/07/2012 at 09:26

Please note that terms and conditions apply. 


\title{
Pulsed performance of pyroelectric detectors
}

\author{
Efthymiou S and Ozanyan K B \\ School of Electrical and Electronic Engineering, \\ The University of Manchester, Manchester M13 9PL, United Kingdom \\ $<$ K.Ozanyan@Manchester.ac.UK>
}

\begin{abstract}
The studies on the behaviour of a Pyroelectric Detector (PED) under the conditions of pulsed load are presented. Taking into account the details of its geometry and thermal connectivity, 3D models resulting to the temperature field in a $\mathrm{LiTaO} 3$ pyroelectric crystal are incorporated in a complete detector. From the timeresolved temperature field, the current resulting from the pyroelectric effect is calculated, followed by the PED output from the I-to- $\mathrm{V}$ converter. The models are verified experimentally with a commercial PED in the case of 1:1 duty cycle and a high electrical time constant. Further, the predicted response to a burst of 2 ns pulses is given for variants of the front-end electronics, allowing the optimal choice of signal processing method. The novelty is the achieved facility to predict the response to pulsed radiation, down to ns pulse widths and arbitrary duty cycle. This is valuable for the engineering of pulsed-source sensor systems requiring detection at room temperature.
\end{abstract}

\section{Introduction}

PEDs are in mass production, most notably because of a sizeable market in the area of security and intruder detection systems[1]. Signal processing from PEDs is presented in literature mainly in terms of their AC response to low-frequency sinusoidal modulation or to square pulses with a 1:1 duty cycle. This approach is unsuitable for developing a signal detection scheme for pulsed detection, e.g. in our case of nanosecond $\mathrm{THz}$ pulses with $\mathrm{kHz}$ frequency, resulting from difference-frequency $\mathrm{THz}$ generation with two near-IR fibre laser/amplifier sources. Thus, in contrast to assuming infinite or insulated boundary constraints as in the current 1D heat transfer models, we obtain the full time-resolved thermal and electrical response to a chosen pulsing strategy, e.g. to bursts of low duty-cycle pulses under a low-frequency envelope. This allows future accurate estimates of the performance of appropriate signal processing methods, such as phase-sensitive detection, depending on the front-end electronic circuit.

\section{PED Thermal Model}

A heat conduction problem, such as heat transferred to a pyroelectric element by laser radiation is not linear, either due to the non-linearity of the differential equation or the boundary conditions or both. Exact solutions of heat conduction problems (with or without time-dependent thermal properties) become extremely difficult or even impossible when complicated boundary conditions are involved. Moreover approximate analytic methods may not be applicable or may not provide the desired degree of accuracy, therefore numerical 
methods are called for. The Finite Difference Method (FDM) and the Finite Element Method (FEM) are widely used to solve both transient and steady-state heat conduction problems and are available as standard software packages.

Modelling the thermal behaviour of any thermal detector requires assumptions about the absorption and reflection coefficients, and justification for neglecting the heat transfer in a three dimensional space. Moreover, the timing response of the detector, which is of main interest in this work, is strongly depended on its particular engineering design, in terms of materials and geometry. The temperature of such bodies can be taken to be a function of time only, $\mathrm{T}(\mathrm{t})$. Heat transfer analysis, utilizing the "lumped" approach, considers a uniform internal temperature distribution at all times $\mathrm{T}(\mathrm{t})$, which would be justified for simple cases. A simplified "lumped" thermal model of the PED can be approximately represented using an equivalent electrical RC network[2,3].
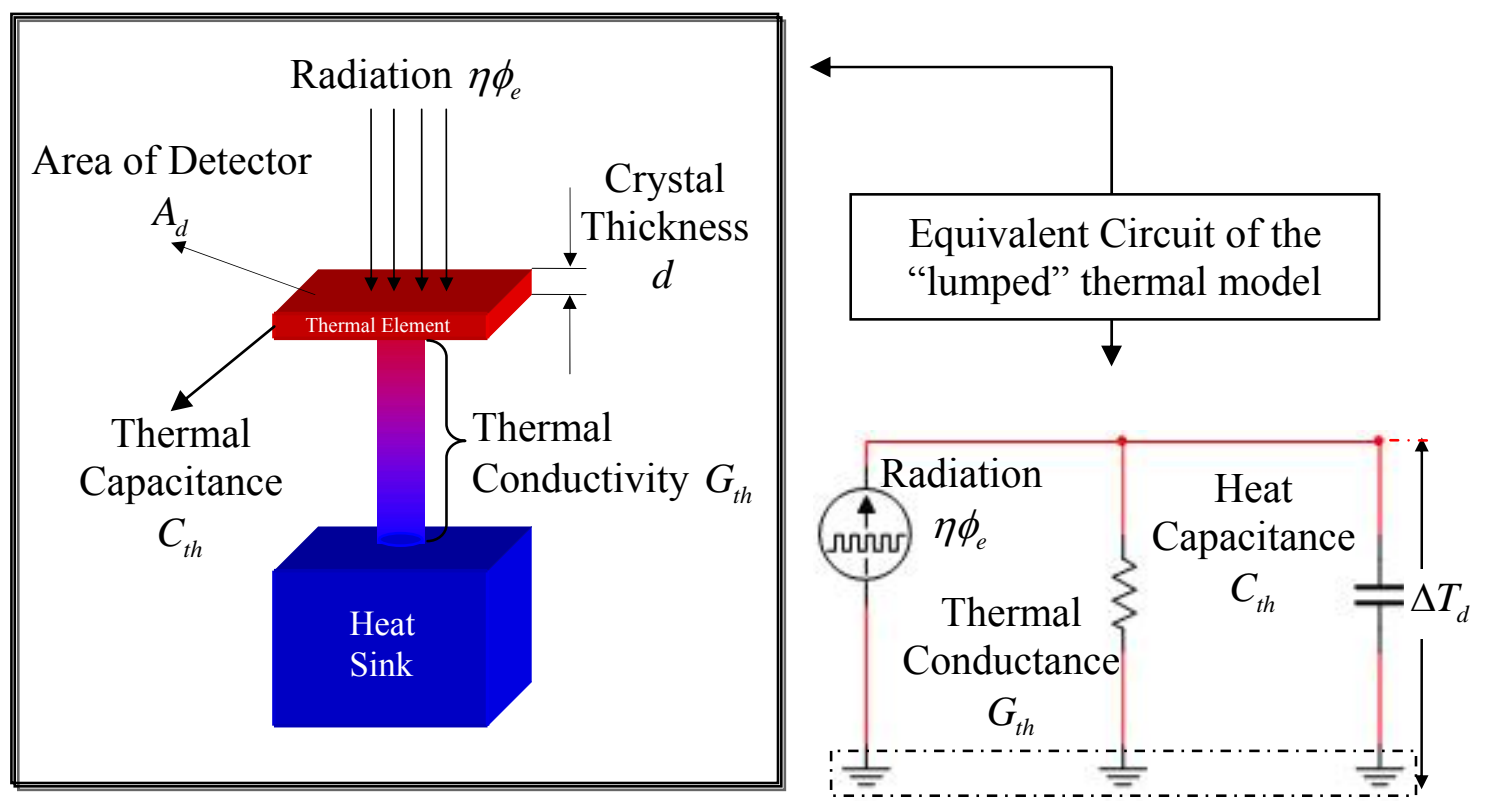

Figure 1 Simplified lumped thermal model with the equivalent "heat circuit" using equivalent electrical components

Considering the heat balance equation

$$
C_{t h} \frac{d \Delta T_{d}(t)}{d t}+G_{t h} \Delta T_{d}(t)=\eta \phi_{e}
$$

The values of the equivalent RC electrical components shown in Fig. 1 can be used to describe the thermal properties (radiation, heat capacity and thermal conductivity). In the less ideal case shown in Fig.2, where the sensitive thermal element consists of several layers, its thermal properties are defined by the resulting values of conductance and heat capacitance, $G t h$ and $C t h$ respectively:

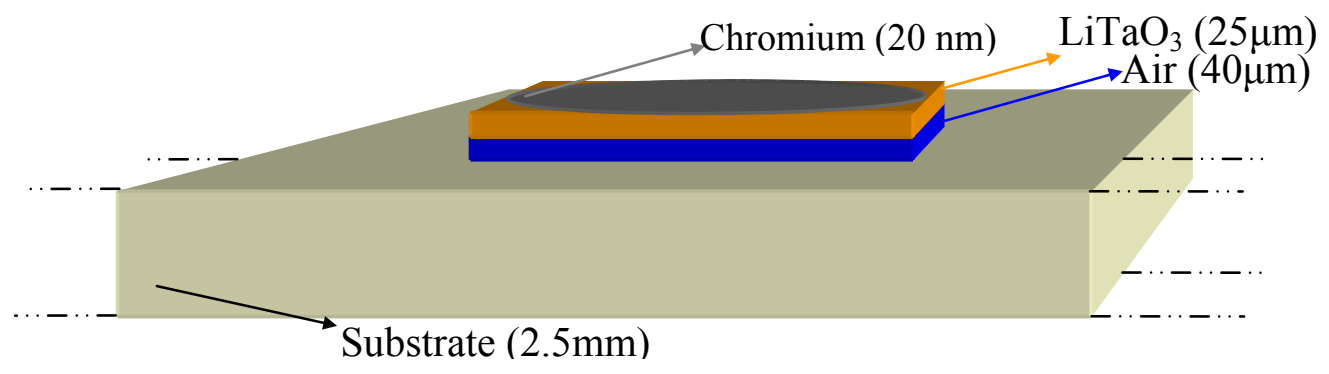

Figure 2 Illustration of the layer structure of the PED SPH-43 from Spectrum Detectors 


$$
\begin{gathered}
G_{\text {th }}=A_{d}\left(\frac{k_{\text {chromium }}}{d_{\text {chromium }}}+\frac{k_{\text {crystal }}}{d_{\text {crystal }}}+\frac{k_{\text {air }}}{d_{\text {air }}}+\frac{k_{\text {substrate }}}{d_{\text {substrate }}}\right) \\
C_{\text {th }}=A_{d}\left(\rho_{\text {chrom }} C_{\text {chrom }} d_{\text {chrom }}+\rho_{\text {cryst }} C_{\text {cryst }} d_{\text {cryst }}+\rho_{\text {air }} C_{\text {air }} d_{\text {air }}+\rho_{\text {subst }} C_{\text {subst }} d_{\text {subst }}\right)
\end{gathered}
$$

Equation (1.1) can therefore be solved (in Laplace or time domain) and the constants Gth and Cth can be calculated using Equations (1.2) and (1.3).

The pyroelectric crystal of the commercial PED SPH-43 has thickness of $25 \mu \mathrm{m}$ and is mounted on a ceramic (usually Alumina) in a bridge configuration as shown in Figure 3 . The air gap between the crystal and the ceramic thermally isolates the crystal providing a longer thermal time constant. The top surface of the crystal is covered with a partially absorbing metal electrode (typically Chromium $\sim 20 \mathrm{~nm}$ ) and the bottom surface is covered again by Chromium, followed by a thin layer of gold with $\sim 40 \mathrm{~nm}$ combined thickness. A small diameter $(0.0762 \mathrm{~mm})$ gold wire is attached to the top electrode and in turn to the header pin. The electric contact to the back of the crystal is made by conductive epoxy that runs to the header pin.

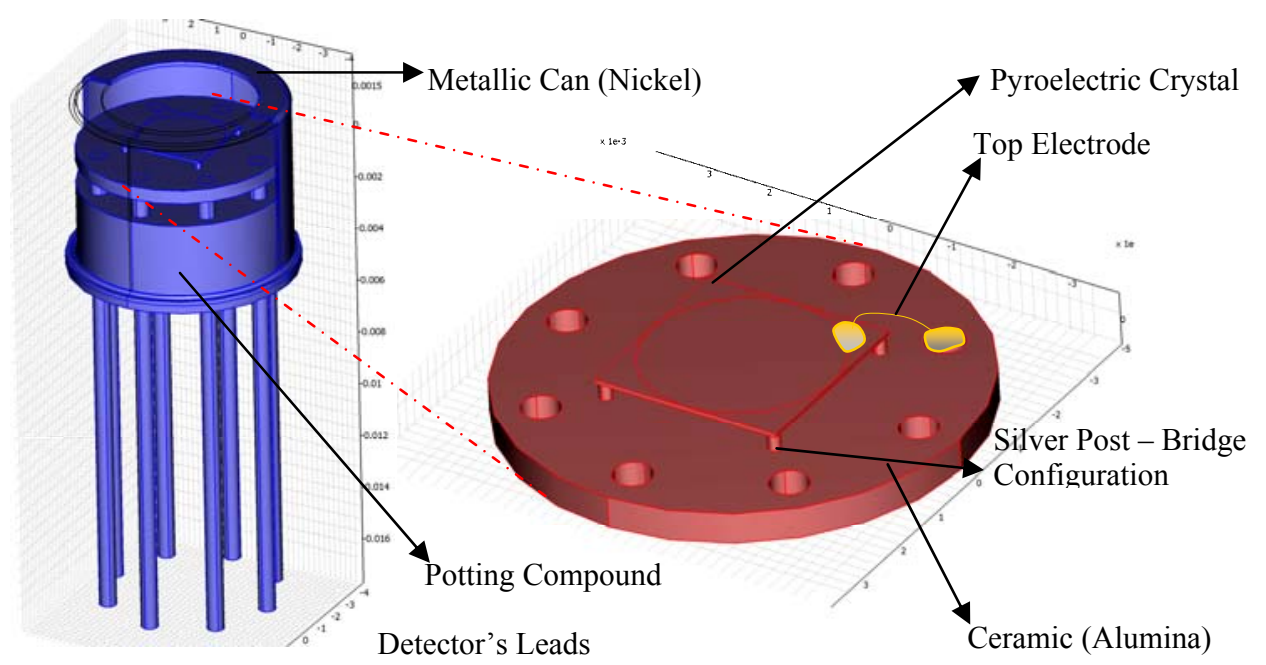

Figure 3 Three dimensional representation of the SPH-43 PED from Spectrum Detectors

Due to the complexity of the detector's geometry, which is ignored in the lumped approach, values of $G_{t h}$ and $C_{t h}$ will yield a less accurate voltage response in terms of timing as well as gain. Using numerical methods, such as FEM or FDM, the success of the model depends on relevance of the defined boundary conditions. A particular PED, SPH-43 from Spectrum Detector has been modelled by using FEM within the COMSOL Multiphysics software.

\section{Thermal to Electrical Conversion}

Following from the pyroelectric properties[4] of the crystal the pyroelectric current is proportional to the rate of polarization change with temperature known as the pyroelectric coefficient $p$, the area $A_{d}$ and the rate of temperature change $\mathrm{d} \Delta \mathrm{T}_{\mathrm{d}} / \mathrm{dt}$ within the crystal[5], as shown in Equation (1.4),

$$
I_{\text {Pyro }}=\frac{d P}{d T} \times \frac{d \Delta T_{d}}{d t} A_{d}=p A_{d} \frac{d \Delta T_{d}}{d t}
$$

The total pyroelectric current is the sum of the contributions (1.4) from each volume element (voxel) calculated from the temperature distribution on a regular 3D grid, exported from COMSOL.

\section{Electrical Conversion}

A PED can be considered as a very high-impedance, low-current source. A voltage signal is obtained as the output of an (I-V) converter integrated within a Spectrum Detector package. 
This is achieved in two modes; the Current Mode (CM) and Voltage Mode (VM) as Fig. 4 depicts.

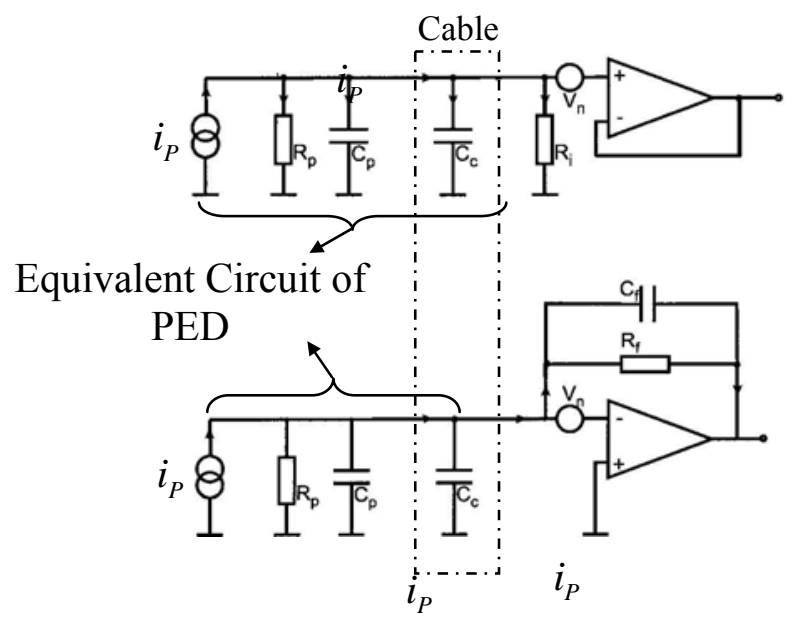

(a)

(b)

Figure 4 Circuit diagrams of (a) Voltage Mode and (b) Current Mode

The transfer function of each mode is shown in the following Equations (1.5) and (1.6):

Current Mode:

$$
\frac{V_{\text {out }}^{C M}}{i_{P}}=\frac{-R_{f}}{1+s R_{f} C_{f}}=\frac{-R_{f}}{1+s \tau_{e l}^{C M}}
$$

Voltage Mode:

$$
\frac{V_{\text {out }}^{V M}}{i_{P}}=\frac{R_{e}}{\left(1+s R_{e} C_{e}\right)}=\frac{R_{e}}{\left(1+s \tau_{e l}^{V M}\right)}
$$

Where $R_{e}=\frac{R_{D} R_{i}}{R_{D}+R_{i}}, \quad C_{e}=C_{D}+C_{c}, \quad R_{e} C_{e}=\tau_{e l}^{V M} \quad$ and $\quad R_{f} C_{f}=\tau_{e l}^{C M}$

The SPH-43 is a current mode device utilizing a transimpedance amplifier. A high value feedback resistor $(100 \mathrm{G} \Omega$ ) is used to achieve high gain. Using the equivalent transfer function of the noise analysis of the I-V converter Simulink/Matlab can be derived. Alternatively, National Instrument (NI) Multisim is a very powerful simulator based on PSpice, where a large library of electronic components (with non-ideal characteristics) is available. This allows convenient simulation of the electrical part of the PED as well as advises the design of further electronic circuits connected to the output of the detector.

\section{Results}

A 3D heat transfer model of the PED is constructed in COMSOL Multiphysics to determine its thermal response to a wide range of irradiation scenarios. The time-dependent temperature field (illustrated in Fig. 5 at a fixed time) is obtained for the $\mathrm{LiTaO}_{3}$ pyroelectric crystal, as a part of a complete, commercially available PED. The pyroelectric current is obtained from the time derivative of the temperature field and is further presented to a I-V Converter, to yield the electronic output.

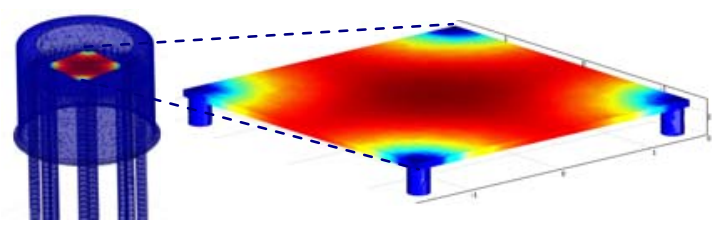

Figure 5 Calculation of the 3D temperature field in the pyroelectric crystal, obtained from a full thermal model of a commercially available PED, illuminated with a 2ns thermal pulse.

The converter circuit (Fig. 6) is simulated under NI Multisim, for the reason stated previously, taking into account an ideal operational amplifier. The complete model is verified 
experimentally by simple experiments based on LED illumination and using the circuit in Fig. $4 \mathrm{~b}$ (current mode).

Fig. 7 shows the voltage response of the PED when illuminated by an LED. The yellow graph shows the voltage response to a step at $\mathrm{t}=0$, where the rate of temperature change $\mathrm{d} \Delta \mathrm{T}_{\mathrm{d}} / \mathrm{dt}$ is obtained using FEM in COMSOL and the I-V conversion simulated in Multisim. The purple graph was solely simulated in Multisim with the "lumped" approach used. The results are all normalised to their maximum value and compared with the experimental results (blue line). While the focus here is on the time response of the detector, the match in absolute amplitude values of voltage is subject to experimental uncertainties in the power delivered to the PED front surface. Overall, the rising part of the response, conditioned by the thermal time-constant, is well reproduced. The departure between the COMSOL-derived model and the experimental data at longer times probably results from parasitic capacitances (the pyroelectric chip, op-amp and connections) that are not accounted for.

Voltage Step Response - Comparison

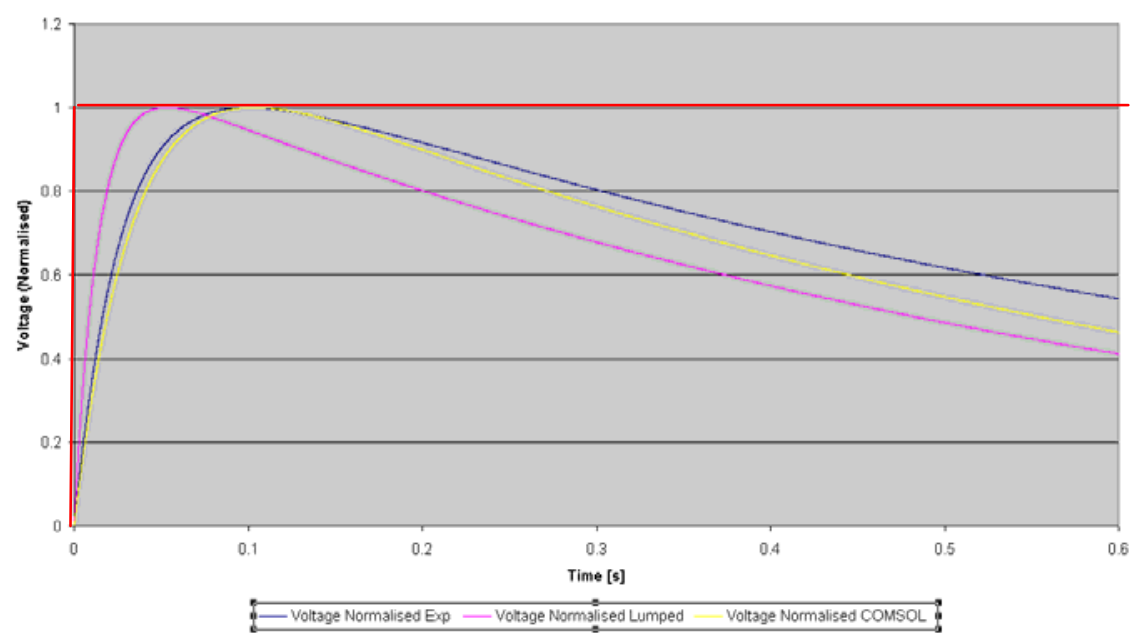

Figure 6 Chart indicating the step response of the PED. Yellow: temperature distribution in the crystal derived in COMSOL, Purple: temperature distribution in the crystal derived by using lumped approach in Multisim, Blue: experimental results

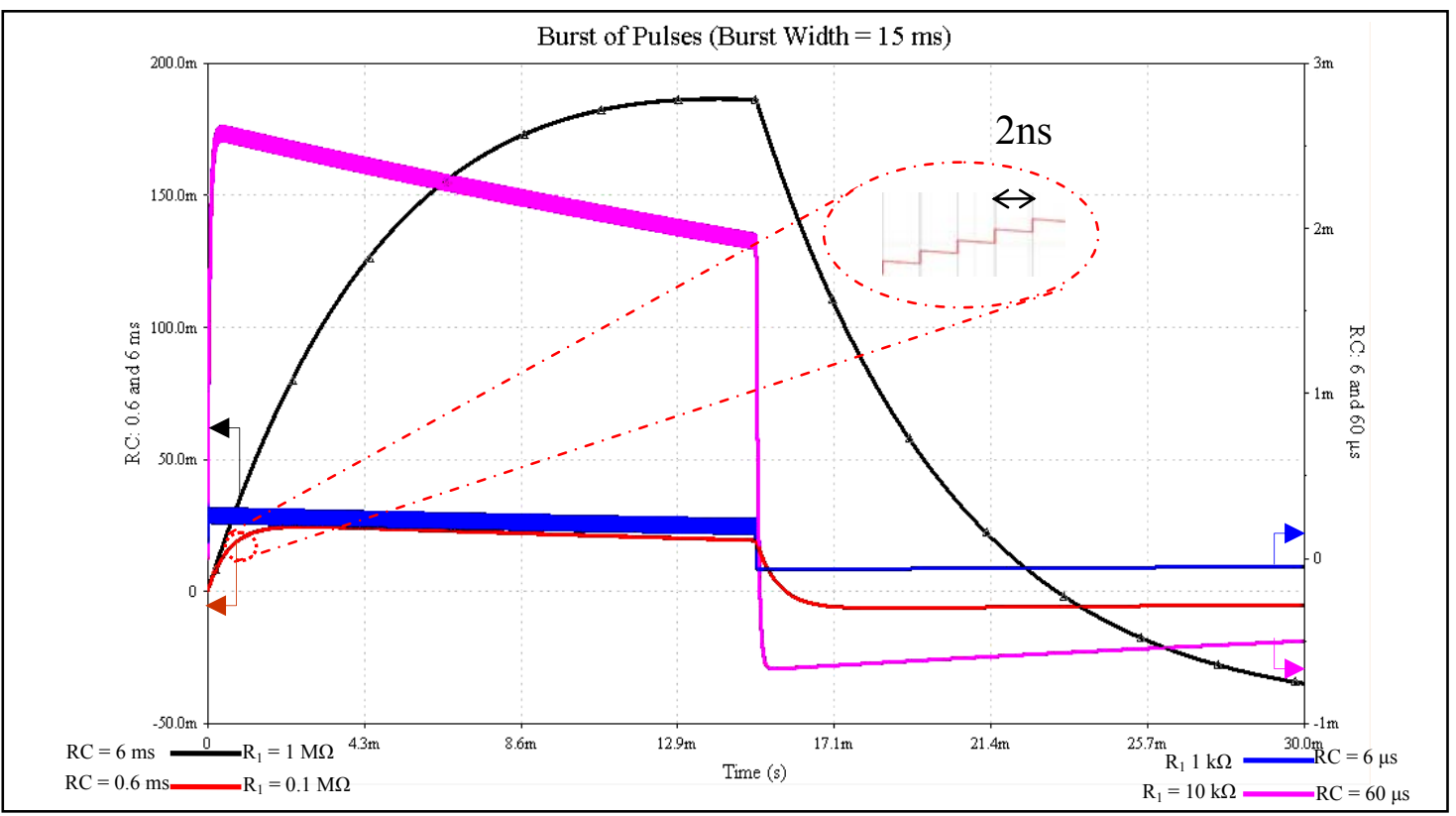

Figure 7 Comparison of the overall detector pulsed response for varying electrical time-constant (varying feedback resistor R1 in Fig.2). The simulations are for a 15ms burst of ns pulses with 1:1000 duty-cycle. 
Further, towards the ultimate goal of modelling of the output signal in the case of a $15 \mathrm{~ms}$ burst of 2 ns pulses with 1:1000 duty cycle, Fig. 8 shows the response for an increasing value of the electrical time constant. A notable gain difference of the low frequency (on/off) envelope responses is observed for each electrical time constant RC. Due to the feedback resistor being integrated within the SPH-43, the output gain of the transimpedance amplifier is limited from above. However it can be reduced, if a smaller electrical time constant is desired, by connecting a shunting resistor. This suggests the importance of the optimal choice of the on/off timing of the burst of ns pulses, directly related to the achievable bandwidth of the envelope and the overall choice of the signal detection scheme.

\section{Conclusions}

A 3D thermal model of a commercial PED (SPH-43) was successfully performed using FEM in COMSOL Multiphysics, to derive the rate of temperature change due to incident pulsed radiation. While the thermal behaviour of the PED could have been simulated using the traditional lumped approach, COMSOL has yielded a more accurate result, by accounting for details such as surface to surface irradiation, convective boundary conditions, etc.. Moreover, COMSOL allows further studies on modelling the thermal behaviour of an array of multiple pyroelectric elements.

PEDs have high performance when operated at low frequencies where the $1 / \mathrm{f}$ noise is dominating.; however this results in a large electrical time constant. Therefore the thermal and electrical time constants will be the key parameters in estimating the optimum shape of the pulse burst sequence to achieve high responsibility and best signal to noise ratio.

\section{References}

[1] R W Whatmore Pyroelectric devices and materials Rep. Prog. Phys. 49 1335-1386 (1986).

[2] V B Samoilov and Y S Yoon, Frequency Response of Multilayer Pyroelectric Sensors IEEE Transactions On Ultrasonics, Ferroelectrics and Frequency Control, vol. 45 (5) , 12551260 (1998).

[3] W P Wheless, Jr. L T Wurtz and J A Wells An Equivalent-Circuit Radiation Sensor Model, IEEE Southeastcon 94 - Creative Technology Transfer: A Global Affair, 1994

[4] S G Porter, A brief guide to pyroelectric detectors, Ferroelectrics, 33 (1) 193-206 (1981).

[5] E L Dereniak \& G D Boreman , in: Infrared Detectors and Systems, John Wiley, 1996 